\title{
Intervenções para elevar a adesão dos profissionais de saúde à higiene de mãos: revisão integrativa
}

Interventions to improve the compliance of health care professionals to hand washing: an integrative review

Intervenciones para elevar la adhesión de profesionales de salud a la higiene de manos: revisión integrativa

Adriana Cristina de Oliveira ${ }^{1}$, Adriana Oliveira de Paula²

\footnotetext{
${ }^{1}$ Enfermeira, Doutora em Enfermagem. Professora Adjunta da Escola de Enfermagem da Universidade Federal de Minas Gerais (EE/UFMG). Belo Horizonte, MG, Brasil. E-mail: acoliveira@gmail.com.

${ }^{2}$ Enfermeira, Mestre em Enfermagem. Discente do Programa de Pós-Graduação em Enfermagem, nível Doutorado, da EE/UFMG. Belo Horizonte, MG, Brasil.E-mail:bhdedis@yahoo.com.br.
}

\section{RESUMO}

Este estudo teve como objetivo identificar as principais estratégias utilizadas visando melhorar a adesão dos profissionais da saúde à higienização das mãos. Tratou-se de uma revisão integrativa da literatura, com busca de artigos em periódicos de língua inglesa, espanhola e portuguesa. Incluíram-se 23 artigos. Elaborou-se um instrumento eletrônico no Microsoft Office Excel e realizou-se análise descritiva dos principais resultados. 87,1\% dos estudos incluídos apresentou delineamento antes e depois e vários métodos foram utilizados para monitorar as taxas de adesão (observação direta, uso de suprimentos e taxa auto-reportada). 87,0\% dos trabalhos utilizaram intervenções multimodais, sendo as mais empregadas: educação, feedback, disponibilização de álcool e de pôsteres. O grande desafio encontrado se constituiu em não só elevar as taxas de adesão à higiene de mãos, mas, sobretudo, mantê-las elevadas. Observou-se a necessidade de utilizar estratégias multimodais que contribuam para a mudança de comportamento considerando a realidade local.

Descritores: Desinfecção das Mãos; Pessoal de Saúde; Infecção Hospitalar; Enfermagem.

\section{ABSTRACT}

The objective of this study was to identify the main strategies used to improve the compliance of health care professionals to hand washing. This is an integrative literature review, which search included journals in English, Spanish and Portuguese. Twenty-three articles were included. An electronic tool was developed on Microsoft Office Excel and the main results were submitted to descriptive analysis. Of the total studies, $87.1 \%$ had before and after designs and several methods were used to monitor compliance rate (direct observation, supply use and self-reported rates). Multimodal interventions were used in $87.0 \%$, and the most often employed were: education, feedback, alcohol being available and posters. The largest challenge identified was not only improving the compliance rates to hand washing, but, most of all, keeping them high. It was observed there is a need to use multimodal strategies that contribute to behavior change considering the local setting.

Descriptors: Hand Disinfection; Health Personnel; Cross Infection; Nursing.

\section{RESUMEN}

Este estudio objetivó identificar las principales estrategias utilizadas apuntando a mejorar la adhesión de profesionales de salud a la higienización de manos. Revisión integrativa de literatura, con búsqueda en publicaciones de lengua inglesa, española y portuguesa. Se incluyeron 23 artículos. Se elaboró un instrumento electrónico en Microsoft Office Excel y se realizó análisis descriptivo de los principales resultados. El 87,1\% de los estudios presentó delineamiento antes y después, y varios métodos fueron utilizados para monitorear las tasas de adhesión (observación directa, uso de suplementos y tasa auto-reportada). $87,0 \%$ de los trabajos utilizaron intervenciones multimodales, prevaleciendo: educación, feedback, disponibilización de alcohol y de pósteres. El gran desafío encontrado se constituyó en no solamente elevar las tasas de adhesión a la higiene de manos, sino también en mantenerlas elevadas. Se observó necesidad de utilizar estrategias multimodales que contribuyan al cambio de comportamiento, considerando la realidad local.

Descriptores: Desinfección de las Manos; Personal de Salud; Infección Hospitalaria; Enfermería. 


\section{INTRODUÇÃO}

A higienização das mãos (HM) se destaca como uma das medidas mais importantes para o controle das infecções relacionadas à assistência em saúde (IRAS). Foi de forma pioneira instituída há mais de 150 anos e ainda hoje é avaliada como de relevância e eficácia, seja pela sua praticidade, baixo custo e, sobretudo, custo-benefício para prevenção e controle das IRAS ${ }^{(1)}$.

A possibilidade de transmissão de microrganismos pelas mãos dos profissionais de saúde, a eficácia da higienização das mãos na redução da carga microbiana das mãos dos profissionais assistenciais e, ainda, a redução das taxas globais de infecção após a adoção de medidas de higienização das mãos tem sido registrada(24).

Pesquisas ${ }^{(5-7)}$ que avaliam o conhecimento e atitude dos profissionais da área da saúde revelam que estes estão cientes da importância da HM no controle de doenças transmissíveis e sobre os momentos em que a higienização das mãos deve ser realizada. Entretanto, é observado um distanciamento entre a teoria e a prática, uma vez que as taxas de adesão à higienização das mãos são baixas, tanto nacionalmente quanto internacionalmente e raramente ultrapassam a $50 \%{ }^{(5-7)}$.

Diante disso, as agências nacionais e internacionais de saúde, tais como a Organização Mundial de Saúde (OMS), o Center for Disease Control and Prevention (CDC) e a Agência Nacional de Vigilância em Saúde (ANVISA), vêm promovendo campanhas no sentido de propor estratégias que visem influenciar a melhoria da adesão à higienização das mãos entre os profissionais da saúde ${ }^{(1-}$ 2,8).

A exemplo disto pode-se citar a campanha "Safe lives: clean your hands" (Salve vidas: lave suas mãos), pertencente ao programa "Clean Care is Safer Care" (Cuidado Limpo é Cuidado Seguro), proposta em 2009 pela OMS. Para tanto, foi sugerida a estratégia "My five moments for hand hygiene" (Meus Cinco Momentos para Higienização das Mãos) apontando de forma resumida as principais oportunidades de higienização das mãos para os profissionais da saúde, durante o cuidado assistencial (antes do contato com o paciente, antes de realizar procedimento, após contato com fluidos corpóreos, após tocar o paciente e tocar superfícies próximas ao paciente) $)^{(1)}$.
Baseado nas baixas taxas de adesão à HM por parte dos profissionais e nas recomendações das agências de saúde, várias iniciativas vêm sendo desenvolvidas por controladores de infecção com o intuito de elevar a adesão a tal medida de prevenção(7,9-10). Contudo, há pouca informação sistematizada sobre as intervenções adotadas e os resultados encontrados.

Diante disto, este estudo teve como objetivo identificar as principais estratégias utilizadas visando melhorar a adesão dos profissionais da saúde à higienização das mãos.

\section{MÉTODOS}

Trata-se de uma revisão integrativa da literatura, cuja finalidade foi reunir e sintetizar evidências disponíveis em artigos originais produzidos sobre o tema Melhoria da adesão de profissionais de saúde à higienização das mãos. Como pergunta norteadora definiu-se: Quais são as intervenções que vem sendo utilizadas visando melhorar a adesão dos profissionais de saúde à higienização das mãos?

A busca de artigos foi realizada em periódicos de língua inglesa, espanhola e portuguesa, por meio nos portais da Coordenação de Aperfeiçoamento de Pessoal de Nível Superior (CAPES), da Biblioteca Virtual em Saúde (BVS) e da Biblioteca Nacional de Medicina (PubMed), cujas bases de dados foram: Literatura Latino-Americana e do Caribe em Ciências da Saúde (LILACS), a Bibliografia Médica (MEDLINE®), Biblioteca Científica Eletrônica Virtual (SciELO), Science Direct, Isi Web of Knowlegde e SCOPUS.

Foram selecionados os manuscritos publicados após o "Guia de implementação da estratégia multimodal da OMS" de 2009. Os seguintes descritores, de acordo com os descritores em ciências da saúde (DECS) e o Medical Subject Headings (MESH), respectivamente, foram utilizados para a busca dos artigos: lavagem de mãos/handwashing, estudos de intervenção/intervention studies.

Consideraram-se como critérios de inclusão: ser um estudo original, ter como objetivo testar alguma intervenção para a melhoria da adesão à higienização das mãos durante o cuidado assistencial e ter sido aplicado em profissionais da área da saúde.

Foram recuperados 131 trabalhos de acordo com os descritores utilizados. Após a leitura dos títulos e resumos observou-se que 23 artigos abordavam o tema 
de interesse e atendiam aos critérios de inclusão sendo considerados para análise. Alguns artigos apareceram em mais de uma base de dados, sendo contabilizados apenas uma vez.

Para avaliação dos artigos selecionados e a realização de uma coleta sistematizada das informações necessárias, elaborou-se um instrumento eletrônico no Microsoft Office Excel 2007, contendo as seguintes variáveis: título, autores, país, ano, revista, objetivo, desenho do estudo, método utilizado para monitorar as taxas de adesão a HM, momento do cuidado assistencial observado, número de intervenções aplicadas, quais intervenções foram utilizadas, principais resultados, tempo de acompanhamento após o término do período de intervenção e avaliação das taxas de IRAS. Realizou-se análise descritiva dos resultados encontrados nos estudos, utilizando-se o software SPSS versão 19.0 .

\section{RESULTADOS}

A maior parte dos 23 estudos incluídos para análise foi publicada entre 2011 e 2013 (82,5\%). Os países de realização dos trabalhos foram Estados Unidos (21,8\%), seguidos da Espanha (17,5\%), Holanda (13,0\%), Itália e Alemanha (8,8\% cada) e Arábia Saudita, Canadá, Escócia, Kuwait, Suécia, Taiwan e Turquia (4,3\% cada).

Grande parte dos manuscritos apresentavam como desenho metodológico estudos do tipo antes e depois $(87,1 \%)$, sendo que apenas três $(12,9 \%)$ foram do tipo ensaio clínico randomizado. Somente $21,7 \%$ dos estudos tiveram grupo controle.

Os trabalhos selecionados empregaram diferentes métodos para avaliar as taxas de adesão dos profissionais à $\mathrm{HM}$, sendo que, dentre estes métodos, a observação direta mereceu destaque, uma vez que $91,3 \%$ dos trabalhos a utilizaram. Os outros métodos abordados foram o consumo de suprimentos $(17,2 \%)$ e taxa auto reportada (8,7\%). Ressalta-se que quatro $(17,2 \%)$ artigos utilizaram mais de um método (observação e consumo de suprimentos ou observação e taxa auto-reportada) de avaliação da taxa de adesão à HM concomitantemente e um (4,3\%) artigos não deixaram claro o método utilizado.

Levando-se em consideração que a maior parte dos estudos utilizou o método de observação direta para avaliar a taxa de adesão à HM, foi analisado também o momento da assistência ao paciente em que foi realizada a observação. Apenas três (13,0\%) estudos realizaram as observações baseadas nos "Meus cinco momentos para a Higienização das Mãos" proposto pela Organização Mundial de Saúde(1). Para o restante dos trabalhos, a maior parte não informou o momento em que a observação foi realizada (34,4\%), observou antes e após o contato com o paciente $(31,1 \%)$, apenas antes do contato com o paciente $(8,7 \%)$, os outros estudos utilizaram métodos não padronizados pela OMS $(4,2 \%)$ ou não se aplica (8,6\%).

No que diz respeito às intervenções, dois $(8,7 \%)$ trabalhos utilizaram apenas uma intervenção, quatro $(17,4 \%)$ utilizaram apenas duas intervenções e o restante $(73,9 \%)$ utilizou três ou mais intervenções simultaneamente.

Dentre as intervenções mais empregadas, destacaram-se educação (78,1\%), feedback (60,8\%), disponibilização de álcool (39,1\%), utilização de pôsteres (34,7\%), envolvimento dos líderes $(21,7 \%)$, mudança da cultura institucional $(21,7 \%)$, envolvimento do paciente $(4,3 \%)$ e não informado (8,7\%).

Sobre o tempo de acompanhamento após o término do período de intervenção, houve uma variação de duas semanas, até um ano e meio, sendo que a maior parte dos estudos acompanhou por menos de um ano $(43,4 \%)$ e alguns não deixaram a informação de forma clara $(30,4 \%)$.

Além de acompanhar o impacto da estratégia sobre a adesão a HM, 43,5\% dos estudos avaliaram também as influências das intervenções sobre as taxas de IRAS. Destaca-se que todos os trabalhos reportaram ter encontrado resultados positivos no que diz respeito a melhoria das taxas de adesão à higienização das mãos, mesmo que não obtidos estes resultado em todas as unidades que compuseram a amostra(10-22). Entretanto, houve um estudo que reportou melhoria das taxas de adesão, mas não da técnica utilizada pelos profissionais ${ }^{(14)}$, e outro trabalho que relatou não ter obtido redução das infecções relacionadas à assistência em saúde ${ }^{(12)}$

O Quadro 1 apresenta uma síntese das principais características dos trabalhos incluídos na análise, indicando o país de realização, o método utilizado de monitorização das taxas de adesão e as intervenções utilizadas. 
Quadro 1: Síntese das principais características dos trabalhos incluídos na análise: país, ano, método de monitorização das taxas de adesão e intervenções utilizadas.

\begin{tabular}{|c|c|c|c|}
\hline País/Ano & Método & Intervenções & Principais resultados \\
\hline Espanha/2009(4) & TAR & Educação & $\begin{array}{l}\text { Houve aumento da taxa de adesão a HM por 6- } \\
9 \text { meses após a intervenção e diminuição das } \\
\text { de infecção nos primeiros dois meses. }\end{array}$ \\
\hline Itália/2009(10) & OD & $\begin{array}{c}\text { Não deixa claro quais intervenções } \\
\text { foram utilizadas, apenas declarando } \\
\text { ter sido adotada uma estratégia } \\
\text { multimodal }\end{array}$ & Eficaz em três das cinco unidades testadas. \\
\hline Holanda/2010 & OD e CS & $\begin{array}{c}\text { Educação, encorajamento de } \\
\text { pessoas chaves para realizar a } \\
\text { técnica correta de HM, mudança de } \\
\text { cultura, feedback. }\end{array}$ & $\begin{array}{l}\text { Melhorou a taxa de adesão a HM e a técnica } \\
\text { utilizada. }\end{array}$ \\
\hline Canadá/2010(12) & OD & Feedback, educação e pôster. & $\begin{array}{l}\text { Elevou as taxas de adesão a HM, mas não } \\
\text { reduziu as taxas de IRAS por MRSA. }\end{array}$ \\
\hline EUA/2011(13) & OD & $\begin{array}{l}\text { Disponibilização de álcool, educação } \\
\text { e feedback. }\end{array}$ & Elevou as taxas de adesão a HM \\
\hline Turquia/2011 (14) & TAR & Treinamento e material escrito & $\begin{array}{c}\text { Melhorou a taxa de adesão, mas não a técnica } \\
\text { utilizada. }\end{array}$ \\
\hline EUA/2011 15$)$ & $\begin{array}{c}\text { Não } \\
\text { informado }\end{array}$ & $\begin{array}{c}\text { Educação e intervenções } \\
\text { comportamentais de reforço }\end{array}$ & $\begin{array}{c}\text { Melhorou no início, mas não foi mantido após } \\
\text { seis meses. }\end{array}$ \\
\hline Itália/2011 (16) & OD & $\begin{array}{l}\text { Feedback, educação, valorização dos } \\
\text { melhores, bottons "ask me", } \\
\text { disponibilização de álcool gel } \\
\text { individual. }\end{array}$ & $\begin{array}{l}\text { Após um ano aumentou a taxa de adesão de } \\
\text { enfermeiros (significativamente) e diminuiu de } \\
\text { médicos (também significativamente) }\end{array}$ \\
\hline Espanha/2011 ${ }^{(17)}$ & OD & $\begin{array}{c}\text { Aquisição de antisséptico, } \\
\text { Informação sobre o antisséptico e } \\
\text { cartazes informativos. }\end{array}$ & $\begin{array}{l}\text { Elevou as taxas de adesão a HM por seis meses } \\
\text { mas não houve melhoria na técnica utilizada. }\end{array}$ \\
\hline EUA/2011 ${ }^{(18)}$ & OD & $\begin{array}{c}\text { Métodos de melhoria da qualidade, } \\
\text { apoio da liderança, treinamento, } \\
\text { feedback. }\end{array}$ & Elevou as taxas de adesão a HM \\
\hline Taiwan/2011 ${ }^{(19)}$ & OD & $\begin{array}{c}\text { Treinamento, feedback, } \\
\text { disponibilização de álcool, multas } \\
\text { para profissionais com baixa adesão } \\
\text { e incentivo financeiro para unidades } \\
\text { com maiores taxas de adesão }\end{array}$ & $\begin{array}{c}\text { Obteve melhoria das taxas de adesão à HM } \\
\text { entre todas as categorias profissionais, } \\
\text { aumento do consumo de álcool, houve uma } \\
\text { tendência decrescente nas taxas de IRAS de } \\
\text { forma global. }\end{array}$ \\
\hline Alemanha/2012(20) & OD & $\begin{array}{l}\text { Treinamento, feedback individual e } \\
\text { coletivo e disponibilização de álcool }\end{array}$ & $\begin{array}{c}\text { Obteve aumento do número de ações } \\
\text { realizadas, paralelamente a um declínio do } \\
\text { número de oportunidades, aumentando assim } \\
\text { a taxa de adesão à HM. A melhoria mais } \\
\text { significativa ocorreu antes de procedimento } \\
\text { asséptico. }\end{array}$ \\
\hline Holanda/2012(21) & OD e CS & $\begin{array}{l}\text { Lembretes no ambiente de trabalho } \\
\text { (descanso de tela) }\end{array}$ & $\begin{array}{c}\text { Elevou as taxas de adesão à HM, porém só } \\
\text { acompanhou por dois meses após as } \\
\text { intervenções. }\end{array}$ \\
\hline Escócia/2012 (22) & OD e CS & $\begin{array}{l}\text { Feedback imediato associado a } \\
\text { outras intervenções }\end{array}$ & $\begin{array}{c}\text { O feedback esteve associado } \\
\text { significativamente a um aumento das taxas de } \\
\text { adesão à HM. }\end{array}$ \\
\hline Espanha/2012(23) & OD & $\begin{array}{l}\text { Multimodal (algumas das } \\
\text { intervenções: disponibilização de } \\
\text { álcool, feedback, dentre outras). }\end{array}$ & $\begin{array}{c}\text { Estratégias contínuas de melhoria da } \\
\text { qualidade devem ser adicionadas às } \\
\text { estratégias multimodais da OMS }\end{array}$ \\
\hline Kuwaiti/2012 24$)$ & OD & $\begin{array}{c}\text { Lembretes no ambiente de trabalho, } \\
\text { treinamento, feedback e } \\
\text { disponibilização de álcool }\end{array}$ & $\begin{array}{l}\text { Houve melhoria nas taxas de adesão à HM e } \\
\text { redução significativa das taxas de IRAS. }\end{array}$ \\
\hline Espanha/2012(25) & OD e CS & $\begin{array}{l}\text { Treinamentos e lembretes no } \\
\text { ambiente de trabalho }\end{array}$ & $\begin{array}{c}\text { Houve melhoria significativa da taxa de adesão } \\
\text { à HM e do consumo do álcool, entretanto não } \\
\text { houve melhoria significativa para redução de } \\
\text { infecções. }\end{array}$ \\
\hline $\mathrm{EUA} / 2012^{(26)}$ & OD & $\begin{array}{c}\text { Treinamento, feedcabk, } \\
\text { envolvimento de líderes, } \\
\text { disponibilização de álcool e melhoria } \\
\text { de comunicação }\end{array}$ & $\begin{array}{l}\text { Obteve melhoria das taxas de adesão à HM e } \\
\text { redução das taxas de IRAS de forma } \\
\text { estatisticamente significativa. }\end{array}$ \\
\hline
\end{tabular}




\begin{tabular}{|c|c|c|c|}
\hline País/Ano & Método & Intervenções & Principais resultados \\
\hline EUA/2013(27) & OD & $\begin{array}{c}\text { Disponibilização de álcool e } \\
\text { pôsteres informativos }\end{array}$ & Obteve melhoria das taxas de adesão à HM. \\
\hline Alemanha/2013(28) & OD & $\begin{array}{c}\text { Treinamento, feedback e } \\
\text { disponibilização de protocolos }\end{array}$ & $\begin{array}{c}\text { Houve melhoria das taxas de adesão à HM, } \\
\text { redução dos contatos desnecessários, } \\
\text { reduzindo as oportunidades de HM. }\end{array}$ \\
\hline $\begin{array}{c}\text { Arábia } \\
\text { Solanda/2013(29) }\end{array}$ & OD & $\begin{array}{c}\text { Treinamento, feedback, lembretes e } \\
\text { envolvimento de lideres }\end{array}$ & $\begin{array}{c}\text { Houve melhoria das taxas de adesão à HM de } \\
\text { forma significativa, no grupo em que houve } \\
\text { envolvimento de líderes }\end{array}$ \\
\hline Suécia/2013(30) & OD & $\begin{array}{c}\text { Treinamento, feedback e } \\
\text { disponibilização de álcool e } \\
\text { envolvimento de líderes }\end{array}$ & $\begin{array}{c}\text { Obteve melhoria das taxas de adesão à HM e } \\
\text { redução das taxas de IRAS de forma } \\
\text { estatisticamente significativa }\end{array}$ \\
\hline
\end{tabular}

Legenda: O.D. Observação direta; TAR: Taxa auto reportada, CS: consumo de suprimentos

\section{DISCUSSÃO}

A higienização das mãos é considerada a medida mais importante no controle das IRAS. Vem ganhando destaque nos últimos anos, principalmente com as publicações dos guidelines do CDC e da OMS, as quais trouxeram atualizações baseadas em evidências sobre HM, os momentos para sua realização, técnicas e produtos utilizados, dentre outros ${ }^{(1-2)}$.

As evidências a respeito das baixas taxas de adesão à HM levam ao desenvolvimento de pesquisas cujo objetivo é elevar a adesão. Contudo, ensaios clínicos randomizados sobre esta temática são raros, devido à dificuldade de implantar uma metodologia rigorosa e por questões éticas. Assim, são mais comuns os estudos do tipo antes e depois, que utilizam a própria população como grupo controle, em momento anterior à realização das intervenções. A ausência de randomização pode ser vista como uma limitação destes trabalhos, favorecendo a ocorrência de vieses, devido a maior dificuldade de controlar possíveis fatores de confusão(3,16).

Em relação aos métodos de monitorização das taxas de adesão à HM, a OMS indica como padrão-ouro a observação direta, o que justifica a maior parte dos trabalhos adotarem esta metodologia(10-13,16-31). Entretanto, destaca-se que não existe um método ideal de monitorização, sendo que todos possuem vantagens e desvantagens. A observação direta, apesar de ser o único processo capaz de avaliar a técnica de HM, é um método oneroso, que demanda grande quantidade de tempo e sofre alguns vieses, destacando-se dentre eles, o Efeito Hawthorne, ocasionado pela presença do observador no ambiente de trabalho do profissional ${ }^{(1,32)}$.

Dessa forma, outros métodos de monitorização vêm sendo explorados, como a taxa auto-reportada e mensuração de suprimentos utilizados. A taxa autoreportada apresenta como principais vantagens ser menos onerosa e necessitar menos tempo para sua realização, entretanto apresentou baixa validade quando utilizada, devido à possibilidade de os profissionais fornecerem respostas socialmente $\operatorname{aceitas}^{(33)}$. Assim, geralmente as taxas auto-reportadas são menos utilizadas quando comparada aos outros métodos de monitorização das taxas de adesão à HM.

A mensuração de suprimentos permite avaliar a adesão à higienização das mãos de forma global, por todos os profissionais e em todos os turnos de trabalho, contudo, não possibilita identificar quais das oportunidades de HM estão sendo seguidas com maior frequência, qual categoria profissional está aderindo mais às recomendações, ou ainda se a técnica empregada está correta. Esta metodologia está sujeita a diversos tipos de viés, relacionado à quantidade de produtos utilizados por cada profissional ou ainda o uso dos suprimentos por pacientes ou acompanhantes, o que elevaria o consumo de produtos sem, contudo, referir a um aumento das taxas de adesão à $\mathrm{HM}^{(34)}$.

Apesar da maior parte dos artigos analisados ter adotado o método considerado padrão-ouro pela OMS (observação direta), destaca-se que outras diferenças no percurso metodológico devem ser levadas em consideração antes de se realizar qualquer comparação entre os estudos, por exemplo, profissionais observados, horário da observação e, principalmente, o momento do cuidado assistencial observado. Destaca-se uma não padronização da metodologia dos estudos que utilizou observação direta, sendo que uma minoria utilizou os "Cinco momentos da OMS" para realizar as observações, 
o que pode impossibilitar a comparação dos resultados obtidos.

No tocante às intervenções adotadas para elevar as taxas de adesão, destaca-se que várias vêm sendo testadas nas últimas décadas, sendo que estudos mais recentes demonstram que intervenções isoladas mostraram menor efeito em longo prazo, quando comparadas a intervenções multimodais, o que justifica o fato da maioria dos artigos analisados terem utilizado um conjunto de intervenções.

Baseado nisto, a OMS, através do programa estratégias multimodais lançado em 2009, propõe o uso de intervenções relacionadas às mudanças institucionais, educação/treinamento, avaliação e feedback, lembretes no ambiente de trabalho e clima organizacional seguro em conjunto. O objetivo é que até 2020 a cultura de higienização das mãos tenha atingido a excelência, respeitando metas a serem definidas de acordo com a realidade de cada região(1).

As intervenções mais utilizadas pelos artigos analisados foram educação, feedback, disponibilização de álcool e lembretes no ambiente de trabalho. Entretanto, o uso de diversas intervenções concomitantemente torna difícil mensurar o impacto de cada intervenção separadamente ${ }^{(22,29)}$.

A educação tem demonstrado ser eficaz na maior parte dos trabalhos, sendo considerada o melhor método de elevar as taxas de adesão à HM, porque é capaz de transformar conhecimento em comportamento e permite que os participantes retenham a informação(14). Entretanto, não devem ser utilizados métodos conservadores, baseados apenas na transmissão de conhecimento. A educação deve ser inovadora, combinando conceitos teóricos e práticos, envolvendo os profissionais nas atividades, a fim de conseguir melhores resultados(7,11). Quando realizada de forma correta, é reconhecida como medida altamente motivadora para a realização da HM. Entretanto, destaca-se que o único estudo que utilizou tal estratégia de maneira isolada, reportou resultado positivo, utilizando as taxas autoreportadas como método de mensuração da adesão(4).

O uso de pôsteres também tem sido amplamente recomendado, sendo que foi reportado por profissionais como importante incentivo para a realização da HM, funcionando como lembretes no ambiente de trabalho. Destaca-se que os pôsteres devem ser regularmente atualizados e estilos inovadores podem ser utilizados para torná-los mais eficazes e mais fáceis de serem lembrados(21). Em um estudo, o uso de descansos de tela foi adotado, com mudanças a cada duas semanas para chamar a atenção dos profissionais, entretanto, o tempo de acompanhamento foi de apenas duas semanas, não ficando claro o impacto que tal estratégia teria em longo prazo(21).

O feedback também tem demonstrado ser eficaz, principalmente para a manutenção de taxas elevadas de HM. Estudo realizado na Escócia comprovou que o feedback, combinado com outras estratégias, pode contribuir para a manutenção das taxas de adesão à $\mathrm{HM}^{(22)}$.

Estudos testando a disponibilização de álcool se justificam não só pela maior eficácia do álcool demonstrada pela OMS, mas também pelo menor tempo gasto para realização da HM, quando comparado à lavagem das mãos com água e sabão, a qual demanda o tempo do deslocamento do profissional até a pia, a secagem das mãos e o deslocamento de volta ao leito do paciente, além de favorecer o não ressecamento da pele do profissional. A disponibilização de álcool é reconhecida pelos profissionais como um importante fator para elevar a taxa de adesão à HM, contudo, quando utilizada como medida isolada pode não surtir efeito(35).

Tais resultados reforçam a necessidade do uso de estratégias multimodais pra elevar a adesão dos profissionais de saúde à HM. Ainda, ressalta-se que as intervenções devem ser as mais específicas possíveis, voltadas para a realidade de cada local e definidas segundo os motivos relatados pelos profissionais para a não adesão. Devem ser diferenciadas de acordo com a categoria profissional, sendo necessárias intervenções diversas para diferentes categorias ${ }^{(16)}$.

Para tentar garantir uma sustentabilidade de taxas de adesão mais elevadas, as intervenções devem ocorrer em intervalos regulares, além de serem realizados esforços contínuos, incluindo reforço constante do programa de educação, observação rotineira e feedback em níveis individuais e organizacionais(16). Ainda, o suporte administrativo também tem demonstrado ter um impacto positivo na adesão à $\mathrm{HM}^{(26,29-30)}$.

Outra maneira de contribuir para elevar a taxa de adesão à HM seria reduzir a quantidade de oportunidades de HM, ou seja, evitar contatos desnecessários com 
superfícies, mobiliários e até mesmo pacientes. Esta ação pode ser tida como uma estratégia para favorecer a adesão à $H M^{(20,28)}$.

Considera-se como aspectos potenciais para se elevar as taxas de adesão à HM: os fatores predisponentes (conhecimento prévio, atitudes e crenças), a disponibilização de produtos (fornecer mais equipamentos para HM, uso de álcool) e reforço (feedback, motivação positiva e suporte de colegas) ${ }^{(9)}$.

Sobre o tempo de monitorização após a intervenção, observou-se discrepâncias na literatura, sendo que a maior parte dos estudos que observou por período igual ou superior a um ano conseguiu manter a taxa de adesão à HM maior do que a taxa encontrada no período préintervenção, apesar de ter diminuído em relação ao período de intervenção. Tal fato comprova a dificuldade da sustentabilidade de taxas de adesão à HM elevadas, indicando a necessidade de mais estudos sobre o tema e desenvolvimento de novas estratégias de melhoria da adesão à HM.

\section{CONCLUSÃO}

Estudos com o intuito de testar estratégias para elevar as taxas de adesão à higiene de mãos estão se tornando cada vez mais frequentes, justificando a importância do tema e a necessidade de aprimoramentos nas estratégias adotadas.

Intervenções como educação, feedback, disponibilização de álcool e lembretes no ambiente de

\section{REFERÊNCIAS}

1. World Health Organization. WHO guidelines on hand hygiene in health care. First Global Patient Safety Challenge Clean Care is Safer Care [Internet]. Geneva: WHO; 2009 [acesso em: $20 \mathrm{dez}$ 2013]. 270p. Disponível em:

http://whqlibdoc.who.int/publications/2009/9789241597906_e ng.pdf.

2. Boyce JM, Pittet D. Guideline for Hand Hygiene in HealthCare Settings. Recommendations of the Healthcare Infection Control Practices Advisory Committee and the HICPAC/SHEA/APIC/IDSA Hand Hygiene Task Force. MMWR Recomm Rep [Internet]. 2002 [acesso em: 20 dez 2013];51(RR16):1-45. Disponível em:

http://www.cdc.gov/mmwr/PDF/rr/rr5116.pdf.

3. Rosenthal VD, Guzman S, Safdar N. Reduction in nosocomial infection with improved hand hygiene in intensive care units of a tertiary care hospital in Argentina. Am J Infect Control [Internet]. 2005 [acesso em: $20 \mathrm{dez}$ 2013];33(7):392-7. Disponivel em: http://dx.doi.org/10.1016/j.ajic.2004.08.009. 4. Tenías JM, Mayordomo C, Benavent ML, Micó MSF, Esparza MAG, Oriola RA. Impacto de una intervención educativa para promover el lavado de manos y el uso racional de guantes en un trabalho estão entre as mais utilizadas. Apesar de mostrarem ser eficazes em conjunto, nem sempre isoladamente apresentam resultado positivo, indicando a necessidade de intervenções multimodais, quando o assunto é melhoria da adesão à HM. Contudo, ressalta-se que o uso de estratégias multimodais dificulta a avaliação do impacto de cada medida isoladamente, o que justifica a necessidade de mais estudos sobre este aspecto.

Baseado nos estudos encontrados, destaca-se a dificuldade de manter a sustentabilidade das taxas de adesão à $H M$, sendo que esta depende de diversos fatores, inclusive aspectos individuais, comportamentais, culturais, organizacionais, dentre outros, que devem ser levados em consideração durante o planejamento das estratégias a serem empregadas.

Dessa forma, observa-se que elevar a taxa de adesão à higienização das mãos é uma tarefa complexa que extrapola o simples fato de educar os profissionais, sendo necessárias estratégias que contribuam para a mudança de comportamento dos mesmos. Рara que o sucesso seja atingido, deve-se considerar a realidade local, utilizandose medidas específicas para cada instituição. O grande desafio observado neste estudo se constituiu em não só aumentar, mas manter elevadas as taxas de adesão. Para isso, nota-se a necessidade de implantar programas efetivos de educação permanente, monitorização frequente, feedback à equipe multiprofissional, dentre outros, de maneira regular.

hospital comarcal. Rev Calidad Asistencial [Internet]. 2009 [acesso em: 20 dez 2013];24(1):36-41. Disponível em: http://dx.doi.org/10.1016/S1134-282X(09)70074-0.

5. Aiello AE, Malinis M, Knapp JK, Mody L. The influence of knowledge, perceptions, and beliefs, on hand hygiene practices in nursing homes. Am J Infect Control [Internet]. 2009 [acesso em: 20 dez 2013];37(2):164-7. Disponível em: http://dx.doi.org/10.1016/j.ajic.2008.04.258.

6. Eveillard M, Pradelle MT, Lefrancq B, Guilloteau V, Rabjeau A, Kempf $M$, et al. Measurement of hand hygiene compliance and gloving practices in different settings for the elderly considering the location of hand hygiene opportunities during patient care. Am J Infect Control [Internet]. 2011 [acesso em: 20 dez 2013];39(4):339-41. Disponível em: http://dx.doi.org/10.1016/j.ajic.2010.08.023.

7. Neves ZCP, Tipple AFV, Souza ACS, Pereira MS, Melo DS, Ferreira LR. Hand hygiene: the impact of incentive strategies on adherence among healthcare workers from a newborn intensive care unit. Rev Lat Am Enfermagem [Internet]. 2006 [acesso em: 20 dez 2013];14(4):546-52. Disponível em: http://dx.doi.org/10.1590/S0104-11692006000400012. 
8. Agência Nacional de Vigilância Sanitária. Segurança do paciente. Higienização das mãos [Internet]. Brasília: Ministério da Saúde; 2008 [acesso em: 20 dez 2013]. Disponível em: http://www.anvisa.gov.br/servicosaude/manuais/paciente hig maos.pdf.

9. Picheansathian W, Pearson A, Suchaxaya P. The effectiveness of a promotion programme on hand hygiene compliance and nosocomial infections in a neonatal intensive care unit. Int J Nurs Pract [Internet]. 2008 [acesso em: 20 dez 2013];14(4):31521. Disponível em: http://dx.doi.org/10.1111/j.1440172X.2008.00699.x.

10. Saint S, Conti A, Bartoloni A, Virgili G, Mannelli F, Fumagalli $S$, et al. Improving healthcare worker hand hygiene adherence before patient contact: a before-and-after five-unit multimodal intervention in Tuscany. Qual Saf Health Care [Internet]. 2009 [acesso em: 20 dez 2013];18(6):429-33. Disponível em: http://dx.doi.org/10.1136/qshc.2009.032771.

11. Helder OK, Brug J, Looman CW, van Goudoever JB, Kornelisse RF. The impact of an education program on hand hygiene compliance and nosocomial infection incidence in an urban neonatal intensive care unit: an intervention study with before and after comparison. Int J Nurs Stud [Internet]. 2010 [acesso em: 20 dez 2013];47(10):1245-52. Disponível em: http://dx.doi.org/10.1016/j.ijnurstu.2010.03.005.

12. Mertz D, Dafoe N, Walter SD, Brazil K, Loeb M. Effect of a multifaceted intervention on adherence to hand hygiene among healthcare workers: a cluster-randomized trial. Infect Control Hosp Epidemiol [Internet]. 2010 [acesso em: $20 \mathrm{dez}$ 2013];31(11):1170-6. Disponível em: http://dx.doi.org/10.1086/656592.

13. Mayer J, Mooney B, Gundlapalli A, Harbarth S, Stoddard GJ, Rubin MA, et al. Dissemination and sustainability of a hospitalwide hand hygiene program emphasizing positive reinforcement. Infect Control Hosp Epidemiol [Internet]. 2011 [acesso em: 20 dez 2013];32(1):59-66. Disponível em: http://dx.doi.org/10.1086/657666.

14. Erkan T, Findik UY, Tokuc B. Hand-washing behaviour and nurses' knowledge after a training programme. Int J Nurs Pract [Internet]. 2011 [acesso em: $20 \mathrm{dez}$ 2013];17(5):464-9. Disponível em: http://dx.doi.org/10.1111/j.1440172X.2011.01957.x.

15. Harne-Britner S, Allen M, Fowler KA. Improving hand hygiene adherence among nursing staff. J Nurs Care Qual [Internet]. 2011 [acesso em: 20 dez 2013];26(1):39-48. Disponível em: http://dx.doi.org/10.1097/NCQ.0b013e3181e0575f. 16. di Martino P, Ban KM, Bartoloni A, Fowler KE, Saint S, Mannelli F. Assessing the sustainability of hand hygiene adherence prior to patient contact in the emergency department: A 1-year postintervention evaluation. Am J Infect Control [Internet]. 2011 [acesso em: 20 dez 2013];39(1):14-8. Disponível em: http://dx.doi.org/10.1016/j.ajic.2010.06.015. 17. García-Vázquez E, Murcia-Payá J, Allegue JM, Canteras M, Gómez J. Influencia de un programa de intervención múltiple en el cumplimiento de la higiene de manos en una unidad de cuidados intensivos. Med Intensiva [Internet]. 2012 [acesso em: 20 dez 2013];36(2):69-76. Disponível em:

http://dx.doi.org/10.1016/j.medin.2011.07.003. 18. Linam WM, Margolis PA, Atherton H, Connelly BL. Qualityimprovement initiative sustains improvement in pediatric health care worker hand hygiene. Pediatrics [Internet]. 2011 [acesso em: 20 dez 2013];128(3):e689-98. Disponível em:

http://dx.doi.org/10.1542/peds.2010-3587.

19. Chen YC, Sheng WH, Wang JT, Chang SC, Lin HC, Tien KL, et al. Effectiveness and Limitations of Hand Hygiene Promotion on Decreasing Healthcare-Associated Infections. PLoS ONE [Internet]. 2011 [acesso em: 20 dez 2013];6(11): e27163. Disponível em: http://dx.doi.org/10.1371/journal.pone.0027163.
20. Scheithauer S, Eitner F, Mankartz J, Haefner $H$, Nowicki K, Floege $J$, et al. Improving hand hygiene compliance rates in the haemodialysis setting: more than just more hand rubs. Nephrol Dial Transplant [Internet]. 2012 [acesso em: $20 \mathrm{dez}$ 2013];27(2):766-70. Disponível em:

http://dx.doi.org/10.1093/ndt/gfr365.

21. Helder OK, Weggelaar AM, Waarsenburg DC, Looman CW, van Goudoever JB, Brug J, et al. Computer screen saver hand hygiene information curbs a negative trend in hand hygiene behavior. Am J Infect Control [Internet]. 2012 [acesso em: 20 dez 2013];40(10):951-4. Disponivel em:

http://dx.doi.org/10.1016/j.ajic.2011.12.003.

22. Fuller C, Michie S, Savage J, McAteer J, Besser S, Charlett A, et al. The Feedback Intervention Trial (FIT)--improving handhygiene compliance in UK healthcare workers: a stepped wedge cluster randomised controlled trial. PLoS One [Internet]. 2012 [acesso em: 20 dez 2013];7(10):e41617. Disponível em: http://dx.doi.org/10.1371/journal.pone.0041617.

23. Mestre G, Berbel C, Tortajada P, Alarcia M, Coca R, Gallemi G, et al. "The 3/3 Strategy": A Successful Multifaceted Hospital Wide Hand Hygiene Intervention Based on WHO and Continuous Quality Improvement Methodology. PLoS ONE [Internet]. 2012 [acesso em: $20 \mathrm{dez} 2013$ ];7(10): e47200. Disponível em: http://dx.doi.org/10.1371/journal.pone.0047200. 24. Salama MF, Jamal WY, Mousa HA, Al-Abdulghani KA, Rotimi VO. The effect of hand hygiene compliance on hospital-acquired infections in an ICU setting in a Kuwaiti teaching hospital. J Infect Public Health [Intenet]. 2013 [acesso em: 20 dez 2013];6(1):27-34. Disponível em: http://dx.doi.org/10.1016/j.jiph.2012.09.014.

25. Monistrol O, Calbo E, Riera M, Nicolás C, Font R, Freixas N, et al. Impact of a hand hygiene educational programme on hospital-acquired infections in medical wards. Clin Microbiol Infect [Internet]. 2012 [acesso em: 20 dez 2013];18(12):1212-8. Disponível em: http://dx.doi.org/10.1111/j.14690691.2011.03735.x.

26. Kirkland KB, Homa KA, Lasky RA, Ptak JA, Taylor EA, Splaine ME. Impact of a hospital-wide hand hygiene initiative on healthcare-associated infections: results of an interrupted time series. BMJ Qual Saf [Internet]. 2012 [acesso em: $20 \mathrm{dez}$ 2013];21(12):1019-26. Disponível em: http://dx.doi.org/10.1136/bmjqs-2012-000800.

27. Kukanich KS, Kaur R, Freeman LC, Powell DA. Evaluation of a hand hygiene campaign in outpatient health care clinics. Am J Nurs [Internet]. 2013 [acesso em: 20 dez 2013];113(3):36-42 Disponivel em:

http://dx.doi.org/10.1097/01.NAJ.0000427878.80053.f7. 28. Scheithauer S, Kamerseder V, Petersen P, Brokmann JC, Lopez-Gonzalez LA, Mach C, et al. Improving hand hygiene compliance in the emergency department: getting to the point. BMC Infect Dis [Internet]. 2013 [acesso em: $20 \mathrm{dez}$ 2013];13:367. Disponível em: http://dx.doi.org/10.1186/14712334-13-367.

29. Huis A, Schoonhoven L, Grol R, Donders R, Hulscher M, van Achterberg T. Impact of a team and leaders-directed strategy to improve nurses' adherence to hand hygiene guidelines: a cluster randomised trial. Int J Nurs Stud [Internet]. 2013 [acesso em: 20 dez 2013];50(4):464-74. Disponível em:

http://dx.doi.org/10.1016/j.ijnurstu.2012.08.004

30. Al-Tawfiq JA, Abed MS, Al-Yami N, Birrer RB. Promoting and sustaining a hospital-wide, multifaceted hand hygiene program resulted in significant reduction in health care-associated infections. Am J Infect Control [Internet]. 2013 [acesso em: 20 dez 2013];41(6):482-6. Disponível em:

http://dx.doi.org/10.1016/j.ajic.2012.08.009.

31. Mernelius S, Svensson PO, Rensfeldt G, Davidsson E, Isaksson B, Löfgren $S$, et al. Compliance with hygiene guidelines: the effect of a multimodal hygiene intervention and validation 
of direct observations. Am J Infect Control [Internet]. 2013 [acesso em: 20 dez 2013];41(5):e45-8. Disponível em: http://dx.doi.org/10.1016/j.ajic.2012.09.008.

32. Sax H, Allegranzi B, Chraïti MN, Boyce J, Larson E, Pittet D. The World Health Organization hand hygiene observation method. Am J Infect Control [Internet]. 2009 [acesso em: $20 \mathrm{dez}$ 2013];37(10):827-34. Disponível em:

http://dx.doi.org/10.1016/j.ajic.2009.07.003.

33. Moret L, Tequi B, Lombrail P. Should self-assessment methods be used to measure compliance with handwashing recommendations? A study carried out in a French university hospital. Am J Infect Control [Internet]. 2004 [acesso em: $20 \mathrm{dez}$ 2013];32(7):384-90. Disponível em:

http://dx.doi.org/10.1016/j.ajic.2004.02.004.

34. Scheithauer $S$, Haefner $H$, Schwanz $T$, Schulze-Steinen $H$, Schiefer J, Koch A, et al. Compliance with hand hygiene on surgical, medical, and neurologic intensive care units: direct observation versus calculated disinfectant usage. Am J Infect Control [Internet]. 2009 [acesso em: $20 \mathrm{dez}$ 2013];37(10):835-41. Disponivel em: http://dx.doi.org/10.1016/j.ajic.2009.06.005.

35. Haas JP, Larson EL. Impact of wearable alcohol gel dispensers on hand hygiene in an emergency department. Acad Emerg Med [Internet]. 2008 [acesso em: 20 dez 2013];15(4):3936. Disponivel em: http://dx.doi.org/10.1111/j.1553-

2712.2008.00045.x.

Artigo recebido em 30/11/2012.

Aprovado para publicação em 16/09/2013.

Artigo publicado em 31/12/2013. 